\title{
Hybrid quantum Gowdy cosmology: Combining loop and Fock quantizations
}

\author{
M. Martín-Benito, ${ }^{1}$ L. J. Garay, ${ }^{1,2}$ and G. A. Mena Marugán ${ }^{1}$ \\ ${ }^{1}$ Instituto de Estructura de la Materia, CSIC, Serrano 121, 28006 Madrid, Spain \\ ${ }^{2}$ Departamento de Física Teórica II, Universidad Complutense de Madrid, 28040 Madrid, Spain
}

(Received 7 April 2008; published 10 October 2008)

\begin{abstract}
We quantize an inhomogeneous cosmological model using techniques that include polymeric quantization. More explicitly, we construct well-defined operators to represent the constraints and find the physical Hilbert space formed by their solutions, which reproduces the conventional Fock quantization for the inhomogeneities. The initial singularity is resolved in this inhomogeneous model in an extremely simple way and without imposing special boundary conditions, thus ensuring the robustness and generality of this resolution. Furthermore, this quantization constitutes a well-founded step towards the extraction of physical results and consequences from loop quantum cosmology, given the central role of the inhomogeneities in modern cosmology.
\end{abstract}

DOI: 10.1103/PhysRevD.78.083516

PACS numbers: 98.80.Qc, 04.60.Pp, 04.62.+v

Loop quantum cosmology (LQC) [1] has recently undergone important developments. The study of the physical consequences of loop quantum gravity (LQG) [2] for cosmology has motivated the analysis of symmetry reduced models that can be fully quantized by techniques that closely resemble those of LQG [3]. Various homogeneous and isotropic models have been successfully quantized [4]. Other less symmetric systems have been also analyzed [57] with partially satisfactory results.

Any realistic attempt to extract physical results from LQC must consider inhomogeneities, which play a central role in current cosmology. As a most suitable arena to progress in this direction, we will study the quantization of the linearly polarized Gowdy $T^{3}$ model [8]. This family of spacetimes is highly symmetric but preserves one important feature of the full theory: it has an infinite number of degrees of freedom. Its classical solutions are indeed well known and generically present an initial singularity [9]. Besides, its quantization has been addressed for a long time by means of standard nonpolymeric techniques [10] (see also [11] for a related system, namely, the cylindrical waves). More recently, a rigorous Fock quantization of this model has been accomplished, which is shown to be essentially unique [12]. Nonetheless, even this Fock quantization (performed after deparametrizing the system) fails to resolve the cosmological singularity.

To overcome this problem, we will carry out a hybrid quantization, that combines the polymeric quantization characteristic of LQC applied to the homogeneous solutions (which describe Bianchi I spacetimes) with Fock quantization of the inhomogeneities. This approach investigates the effects on quantum geometry underlying LQC only on the homogeneous sector, while it disregards the discreteness of the geometry encoded by the inhomogeneities. A most natural treatment for the inhomogeneities is then the Fock quantization. Indeed, one would expect that a quantum field theory for the inhomogeneities, which can be regarded as a field living on a homogeneous (Bianchi I) background, be approximately valid on the polymerically quantized background. An additional motivation for this approach is the possibility that the inhomogeneities can be finally considered as perturbations. We will represent the quantum constraints of this model, find their solutions, construct the physical Hilbert space, and recover the conventional quantum field theory. To our knowledge this is the first time that this task has been carried out to this level of completion in a system with an infinite number of physical degrees of freedom.

With this aim, we will reduce the classical phase space imposing gauge fixing conditions but, unlike in [12], we will not deparametrize the system completely, so that we will be left with a remnant Hamiltonian constraint, namely, the spatial average of the full Hamiltonian constraint. Its quantum counterpart is not entirely derived from LQG, since the inhomogeneities are not quantized à la loop, but it retains interesting polymeric features. In particular, we will face the problem of polymerically quantizing the internal time. As we will see this may affect the conventional concept of evolution.

We will also analyze the role played by the inhomogeneities in the quantum theory and thus the robustness of the results obtained in homogeneous LQC. In fact, as we have commented, a fundamental motivation for this hybrid quantization is the resolution of the cosmological singularity. The first studies in homogeneous LQC already indicate that the section of constant internal time corresponding to the initial singularity can be avoided in the quantum theory $[3,13]$. In this paper we will show that the loop quantization of the homogeneous degrees of freedom, which correspond to global variables, suffices to "cure" the singularity, without the need to resort to the BKL (Belinsky, Khalatnikov, and Lifshitz) conjecture [14]. Therefore, we do not have to consider all the points individually. We will see that the initial singularity disappears from our quantum theory. Actually, nontrivial physical states contain no contribution corresponding to the singular 
initial section. Furthermore, they do not even evolve to another branch of the Universe with opposite (triad) orientation, and can thus be viewed as arising from "nothing," without imposing any particular boundary condition.

The considered Gowdy model is a globally hyperbolic vacuum spacetime, with spatial sections homeomorphic to a 3-torus and two hypersurface-orthogonal spatial Killing fields [8]. It can be described in terms of coordinates $\{t, \theta, \sigma, \delta\}$ adapted to the symmetries, where $\partial_{\sigma}$ and $\partial_{\delta}$ are the two Killing fields, with $\sigma, \delta \in S^{1}$. The induced spatial 3-metric, the densitized lapse function $\underset{\sim}{N}$ and the shift vector $N^{i}$, with $i \in\{\theta, \sigma, \delta\}$, depend on $t$ and $\theta \in S^{1}$, and are then expandable in Fourier series.

Suitable gauge fixing conditions for the diffeomorphisms generated by the two Killing fields, together with the linear polarization condition, lead to a diagonal 3metric. It can be characterized by three fields that describe the norm of one of the Killing vectors, the area of the isometry group orbits, and the scale factor of the metric induced on the set of group orbits. We further impose that the generator of the conformal transformations of this latter metric and the area of the isometry group orbits be homogeneous functions. These conditions fix the gauge freedom associated to the nonzero Fourier modes of the $\theta$-momentum constraint and of the densitized Hamiltonian constraint, and imply that the functions $N^{\theta}$ and $\underset{\sim}{N}$ are homogeneous [12]. The classically reduced system is then described by three canonical pairs of variables, which correspond to the homogeneous degrees of freedom of a Bianchi I spacetime with $T^{3}$ topology, and by the infinite number of nonzero (inhomogeneous) modes of the field unaffected by the gauge fixing, together with their corresponding conjugate momenta (we adopt the same parametrization as in [12]). We will call them the homogeneous and inhomogeneous sectors, respectively. There remain two constraints: the zero mode of the $\theta$-momentum constraint and the zero mode of the densitized Hamiltonian constraint, which generate $S^{1}$ and time translations.

The homogeneous sector of this reduced Gowdy model is described in Ashtekar variables. We adopt a diagonal gauge for these variables, corresponding to Bianchi I degrees of freedom. Since they can be defined in such a way that they do not depend on the selected fiducial metric [6], we choose the Euclidean one for simplicity. The natural coordinate cell of the system is the $T^{3}$ cell. Then the nontrivial components of the $S U(2)$ gravitational connection and of the densitized triad are $c^{i} /(2 \pi)$ and $p_{i} /\left(4 \pi^{2}\right)$, respectively, with $\left\{c^{i}, p_{j}\right\}=8 \pi G \gamma \delta_{j}^{i}$. Here, $\gamma$ is the Immirzi parameter. We note that $p_{\theta}$ is proportional to the time variable chosen in [12] to deparametrize the system.

The kinematical Hilbert space for the homogeneous sector is constructed adapting LQG procedures to symmetry reduced systems [3,5]. The elementary homogeneous variables are holonomies along edges of oriented coordinate length $2 \pi \mu_{i}$ in the $i$ direction, $\mu_{i}$ being a real number, and triad fluxes through rectangles orthogonal to those directions. The configuration algebra, denoted by $\mathrm{Cyl}_{S}$, is the algebra of almost periodic functions generated by the exponentials $\mathcal{N}_{\mu_{j}}\left(c^{j}\right)=\exp \left(i \mu_{j} c^{j} / 2\right)$ [15], which in the Dirac ket notation become $\left|\mu_{j}\right\rangle$. The homogeneous sector of the kinematical Hilbert space is the Cauchy completion of $\mathrm{Cyl}_{S}$ with respect to the discrete inner product $\left\langle\mu_{i} \mid \mu_{i}^{\prime}\right\rangle=\delta_{\mu_{i} \mu_{i}^{\prime}}$ for each direction. The basis states $\left|\mu_{i}\right\rangle$ are eigenstates of the momentum operator $\hat{p}_{i}$, while the action of $\hat{\mathcal{N}}_{\mu_{i}^{\prime}}$ shifts their label $\mu_{i}$.

Remember that in LQG physical areas are discrete and have a minimum nonzero eigenvalue $\Delta$. This feature has an imprint in LQC. The coordinate length of the holonomy along each edge exhibits a state-dependent minimum value $2 \pi \bar{\mu}_{i}$ [16]. Here, we will adopt the (operator) relation $\bar{\mu}_{i}^{2}\left|\hat{p}_{i}\right|=\Delta$, as proposed in [5] (for additional details on this relation, see [17]). As a result, the operator $\hat{\mathcal{N}}_{\bar{\mu}_{i}}$ generates a state-dependent minimum shift. It is convenient to rename the states by reparametrizing the labels $\mu_{i}$ so that this minimum shift becomes uniform. Carrying out this affine reparametrization [4,5] one introduces, for each direction, a new label $v_{i}\left(\mu_{i}\right)$ such that $\hat{\mathcal{N}}_{\bar{\mu}_{i}}\left|v_{i}\right\rangle=\left|v_{i}+1\right\rangle$ and $\hat{p}_{i}\left|v_{i}\right\rangle=3^{1 / 3} \Delta \operatorname{sgn}\left(v_{i}\right)\left|v_{i}\right|^{2 / 3}\left|v_{i}\right\rangle$. The operators acting on the homogeneous sector can then be written in terms of these elementary ones, which are densely defined in the domain spanned by the states $\left|v_{i}\right\rangle$.

For the inhomogeneous modes of the only field present in the theory, we adopt the Fock quantization of [12]. We represent this field in terms of the basis of $n$-particle states naturally associated with a free massless scalar field [12], $\left|\left\{n_{m}\right\}\right\rangle:=\left|\ldots, n_{-m}, \ldots, n_{m}, \ldots\right\rangle$. Here $m \neq 0$ and $n_{m}<\infty$ is the occupation number of the $m$ th mode. In these states, only a finite set of these occupation numbers differ from zero. The proper Fock subspace annihilated by the constraint that generates $S^{1}$-translations will be called $\mathcal{F}_{p}$. A basis for it is provided by the $n$-particle states that verify the condition $\sum_{m>0} m\left(n_{m}-n_{-m}\right)=0$.

In order to quantize the densitized Hamiltonian constraint of the model, let us first focus on Bianchi I.

LQG techniques provide the (nondensitized) quantum Hamiltonian constraint $\hat{C}_{\mathrm{BI}}$ for Bianchi I in terms of the elementary operators $\hat{p}_{i}$ and $\hat{\mathcal{N}}_{\mu_{i}}[5,17,18]$. We adopt a factor ordering such that this constraint is a sum of terms factorized in each direction, each of these factors being symmetric. Besides, our prescriptions ensure that $\hat{C}_{\mathrm{BI}}$ annihilates the proper subspace on which any of the operators $\hat{p}_{i}$ vanishes $[17,18]$. Taking into account that the volume operator is $\hat{V}=\sqrt{\left|\hat{p}_{\theta} \hat{p}_{\sigma} \hat{p}_{\delta}\right|}$, we will call it the subspace of zero-volume states. Remarkably, this subspace decouples when the constraint is imposed because, in addition, its complement is invariant under the action of $\hat{C}_{\mathrm{BI}}$. Therefore, we will restrict to this complement in the following. 
We are now ready to write down the densitized quantum Hamiltonian constraint $\hat{\mathcal{C}}_{\mathrm{BI}}$ for Bianchi I. LQG procedures give the regularized inverse volume operator $\widehat{1 / V}$. It only annihilates the zero-volume states, which are removed from our theory. Consequently, its inverse $[\widehat{1 / V}]^{-1}$ is well defined. In principle, Bianchi I physical states (i.e. those annihilated by $\hat{C}_{\mathrm{BI}}$ ) will not be normalizable in the kinematical Hilbert space. These states, denoted by $(\psi \mid$, live in a larger space [19] on which we can define

$$
\hat{\mathcal{C}}_{\mathrm{BI}}=[\widehat{1 / V}]^{-1 / 2} \hat{C}_{\mathrm{BI}}[\widehat{1 / V}]^{-1 / 2}
$$

This operator annihilates the transformed physical states $\left(\tilde{\psi} \mid\right.$, related to $\left(\psi \mid\right.$ by the bijection $\left(\tilde{\psi} \mid=\left(\psi \mid[\widehat{1 / V}]^{1 / 2}\right.\right.$.

In the full Gowdy model, once the homogeneity of the densitized lapse function is taken into account, the densitization of the Hamiltonian constraint is carried out by means of the transformation explained above. Again, states on the kernel of any of the $\hat{p}_{i}$ 's are decoupled. The densitized Hamiltonian constraint $\hat{\mathcal{C}}_{\mathrm{G}}$ is the sum of two terms: the already quantized Bianchi I part and the part that involves the inhomogeneities. The latter depends on the absolute value of our internal time variable, $\left|p_{\theta}\right|$. In particular, there is a term quadratic in the inverse of its square root, for which LQC provides the corresponding regularized operator (which acts diagonally on the states $\left|v_{\theta}\right\rangle$ ). In addition, this part of the constraint also depends on the connections, which are not well defined operators in LQC. This difficulty can be overcome if we notice that the only dependence on the connections occurs through factors of the form $c^{i} p_{i}$. Indeed, if we compare $\hat{\mathcal{C}}_{\mathrm{BI}}$ with its classical counterpart, it is possible to identify a natural quantum prescription to represent $c^{i} p_{i}$. The corresponding symmetric operator, $\hat{\Theta}_{i}$, can be written in terms of the operators $\left|\hat{p}_{i}\right|, \operatorname{sgn}\left(\hat{p}_{i}\right)$, and $\hat{\mathcal{N}}_{ \pm 2 \bar{\mu}_{i}}$. It can be shown to be essentially self-adjoint. Its action on the states $\left|v_{i}\right\rangle$ is of the form $[17,18]$

$$
\hat{\Theta}_{i}\left|v_{i}\right\rangle=-i \pi \gamma l_{\mathrm{p}}^{2}\left[f_{+}\left(v_{i}\right)\left|v_{i}+2\right\rangle-f_{-}\left(v_{i}\right)\left|v_{i}-2\right\rangle\right],
$$

where $l_{\mathrm{p}}=\sqrt{G \hbar}$ is the Planck length and the functions $f_{+}\left(v_{i}\right)$ and $f_{-}\left(v_{i}\right)$ possess the relevant property that they vanish in the intervals $[-2,0]$ and $[0,2]$, respectively.

The explicit expression of the constraint $\hat{\mathcal{C}}_{\mathrm{G}}$ is [18]

$$
\begin{aligned}
\hat{\mathcal{C}}_{\mathrm{G}}= & -\frac{2}{\gamma^{2}}\left[\hat{\Theta}_{\theta} \hat{\Theta}_{\delta}+\hat{\Theta}_{\theta} \hat{\Theta}_{\sigma}+\hat{\Theta}_{\sigma} \hat{\Theta}_{\delta}\right] \\
& +l_{\mathrm{p}}^{2}\left[\frac{\left(\hat{\Theta}_{\sigma}+\hat{\Theta}_{\delta}\right)^{2}}{\gamma^{2}}\left(\frac{\widehat{1}}{\sqrt{\left|p_{\theta}\right|}}\right)^{2} \hat{H}_{\mathrm{int}}^{\xi}+32 \pi^{2}\left|\hat{p}_{\theta}\right| \hat{H}_{0}^{\xi}\right], \\
\hat{H}_{\text {int }}^{\xi}= & \sum_{m \neq 0} \frac{1}{2|m|}\left[2 \hat{a}_{m}^{\dagger} \hat{a}_{m}+\hat{a}_{m}^{\dagger} \hat{a}_{-m}^{\dagger}+\hat{a}_{m} \hat{a}_{-m}\right] .
\end{aligned}
$$

Here, $\hat{H}_{0}^{\xi}=\sum_{m}|m| \hat{a}_{m}^{\dagger} \hat{a}_{m}$ is the Hamiltonian of a massless free scalar field, and $\hat{a}_{m}$ and $\hat{a}_{m}^{\dagger}$ are the annihilation and creation operators of particles in the $m$ th mode corresponding to that field, such that $\left[\hat{a}_{m}, \hat{a}_{\tilde{m}}^{\dagger}\right]=\delta_{m \tilde{m}}$. In the above formula for $\hat{\mathcal{C}}_{\mathrm{G}}$, the first line is the densitized Hamiltonian constraint $\hat{\mathcal{C}}_{\mathrm{BI}}$ of Bianchi I.

Since $\hat{\Theta}_{i}$ is a difference operator and actually it does not relate states $\left|v_{i}\right\rangle$ with $v_{i}>0$ to states with $v_{i}<0$, the label $v_{i}$ can be restricted to any of the semilattices $\mathcal{L}_{\bar{\varepsilon}_{i}}^{ \pm}=\left\{ \pm\left(\varepsilon_{i}+2 k\right), k \in \mathbb{N}\right\}$, where $\varepsilon_{i} \in(0,2]$. Semilattices corresponding to different $\varepsilon_{i}$ or different signs do not get mixed under the action of the constraint. In this sense the homogeneous sector is superselected. We can then focus our study on the kinematical Hilbert space $\left(\otimes_{i} \mathcal{H}_{\varepsilon_{i}}\right) \otimes \mathcal{F}_{p}$, where $\mathcal{H}_{\varepsilon_{i}}$ is the completion of the span of states $\left|v_{i}\right\rangle$ with $v_{i}$ in the specific semilattice $\mathcal{L}_{\varepsilon_{i}}^{+}$.

Note also that $\hat{\Theta}_{\sigma}$ and $\hat{\Theta}_{\delta}$ are Dirac observables. A careful analysis, complemented with numerical evidence, supports that the spectrum of $\hat{\Theta}_{i}$ is absolutely continuous and coincides with the real line [17]. Therefore, its generalized eigenstates $\left|\lambda_{i}\right\rangle$ are delta-normalizable with respect to the Lebesgue measure $d \lambda_{i}$.

The constraint $\hat{\mathcal{C}}_{\mathrm{G}}$ is a well-defined operator in the domain spanned by $\left\{\left|v_{\theta}\right\rangle \otimes\left|v_{\sigma}\right\rangle \otimes\left|v_{\delta}\right\rangle \otimes\left|\left\{n_{m}\right\}\right\rangle ; v_{i} \neq 0\right\}$ [20]. This result shows the viability of combining the polymeric and Fock quantizations. Furthermore, an analysis of the associated deficiency index equation indicates that it possesses no normalizable solutions and therefore that the constraint operator is essentially self-adjoint. Thus, group averaging techniques can in principle be available to determine the space of solutions to the Hamiltonian constraint.

Nonetheless, one can formally obtain the generic expression of the solutions without the need to resort to group averaging, whose application is not immediate in this case. For convenience, we will use the (generalized) eigenstates of $\hat{p}_{\theta}, \hat{\Theta}_{\sigma}$, and $\hat{\Theta}_{\delta}$, instead of those of the $\hat{p}_{i}$ 's, to decompose the homogeneous sector. Then, if we formally expand the states $(\tilde{\psi} \mid$, we can represent them by the coefficients $\left(\tilde{\psi}_{\lambda_{\sigma}, \lambda_{\delta}}\left(v_{\theta}\right)\left|\left\{n_{m}\right\}\right\rangle\right.$. $\hat{\Theta}_{\theta}$ is the only operator in $\hat{\mathcal{C}}_{\mathrm{G}}$ that does not act diagonally on the homogeneous sector. Consequently, the Hamiltonian constraint becomes a recurrence equation that relates the coefficients in three consecutive sections $\left(v_{\theta}-2\right)$, $v_{\theta}$, and $\left(v_{\theta}+2\right)$. However, the coefficient evaluated at $\left(v_{\theta}-2\right)$ appears multiplied by the factor $f_{-}\left(v_{\theta}\right)$. Since $f_{-}\left(\varepsilon_{\theta}\right)=0$, the recurrence equation turns into a consistency relation for the coefficients in the two first sections $\varepsilon_{\theta}$ and $\left(\varepsilon_{\theta}+2\right)$. As a result any coefficient $\left(\tilde{\psi}_{\lambda_{\sigma}, \lambda_{\delta}}\left(v_{\theta}\right)\left|\left\{n_{m}\right\}\right\rangle\right.$ can be expressed in terms of the initial ones $\left(\tilde{\psi}_{\lambda_{\sigma}, \lambda_{\delta}}\left(\varepsilon_{\theta}\right)\left|\left\{n_{\tilde{m}}\right\}\right\rangle\right.$. The formal solution of the constraint is, obviating the $\lambda$ dependence and the projection on $n$-particle states, 


$$
\begin{aligned}
\left(\tilde{\psi}\left(\varepsilon_{\theta}+2 k\right) \mid=\right. & \left(\tilde{\psi}\left(\varepsilon_{\theta}\right) \mid \sum_{O(k)}\left[\prod_{\left\{r_{i}\right\}} F\left(\varepsilon_{\theta}+2 r_{i}+2\right)\right]\right. \\
& \times \mathcal{P}\left[\prod_{\left\{s_{j}\right\}} \hat{H}^{\xi}\left(\varepsilon_{\theta}+2 s_{j}\right)\right],
\end{aligned}
$$

where $O(k)$ is the set of possible ways to move from 0 to $k$ by jumps of one or two steps and, for each element of $O(k)$, $\left\{s_{j}\right\}$ is the set of integers followed by a jump of one step, while $\left\{r_{i}\right\}$ are those followed by a jump of two steps [20]. Besides, $F\left(v_{\theta}\right)=f_{-}\left(v_{\theta}\right) / f_{+}\left(v_{\theta}\right)$, the symbol $\mathcal{P}$ denotes path ordering, and $\hat{H}^{\xi}\left(v_{\theta}\right)$ has the form

$$
\hat{H}^{\xi}\left(\boldsymbol{v}_{\theta}\right)=y_{1}\left(\boldsymbol{v}_{\theta}\right)+y_{2}\left(\boldsymbol{v}_{\theta}\right) \hat{H}_{\mathrm{int}}^{\xi}+y_{3}\left(\boldsymbol{v}_{\theta}\right) \hat{H}_{0}^{\xi},
$$

$y_{k}\left(v_{\theta}\right)$ being $\lambda$-dependent functions whose detailed expression is irrelevant for our discussion.

As we have seen, $\left(\tilde{\psi}_{\lambda_{\sigma}, \lambda_{\delta}}\left(\varepsilon_{\theta}\right) \mid\right.$ completely determines the solution, so that we can identify the latter with the corresponding data on the initial section $v_{\theta}=\varepsilon_{\theta}$. To endow the space of these solutions with a Hilbert structure, we choose a complete set of real classical observables and require that their quantum counterparts be self-adjoint operators. At this stage it is easier to relax the $S^{1}$-gauge symmetry, so that we can choose the operators that represent the Fourier sine and cosine coefficients of the nonzero modes as the basic observables for the inhomogeneous sector. For the homogeneous sector, we can select as a complete set of observables (in the $\lambda$ representation) the operators of multiplication by $\lambda_{\sigma}$ and $\lambda_{\delta}$ (i.e., $\hat{\Theta}_{\sigma}$ and $\hat{\Theta}_{\delta}$ ), together with the respective derivatives evaluated on the considered section, $-\left.i \partial_{\lambda_{\sigma}}\right|_{\varepsilon_{\theta}}$ and $-\left.i \partial_{\lambda_{\delta}}\right|_{\varepsilon_{\theta}}$. In order to obtain the physical Hilbert space we first demand reality conditions on all these observables and then restore the $S^{1}$ symmetry. The final result is that, for generic initial data $\left(\tilde{\psi}_{\lambda_{\sigma}, \lambda_{\delta}}\left(\varepsilon_{\theta}\right)\right)$, the physical Hilbert space that one obtains in this way is unitarily equivalent to $L^{2}\left(\mathbb{R}^{2}, d \lambda_{\sigma} d \lambda_{\delta}\right) \otimes \mathcal{F}_{p}$. In particular, let us emphasize that we then recover the Fock quantization of [12] for the physical inhomogeneous sector.

Several comments are in order once we have completed the quantization of this Gowdy model.

We remark that the general expression of the solutions is only formal. A more rigorous interpretation in terms of structures associated with the kinematical Hilbert space is obstructed by the production of infinite particle pairs caused by the operator $\hat{H}_{\text {int }}^{\xi}$ and by the $v_{\theta}$ dependence of the inhomogeneous part of the constraint, which prevents its diagonalization in terms of particle states with a fixed mass for all the $v_{\theta}$ sections. Nevertheless, we can in fact make full sense of the solutions in certain situations.

This is the case if one introduces a cutoff for the wave number of the field modes, which can be understood as a further symmetry reduction of the Gowdy model. Solutions to the constraint are then in the algebraic dual of the dense set of $n$-particle states on each $v_{\theta}$ section. Actually, the mentioned physical Hilbert space for the full Gowdy model can then be attained in the limit in which the cutoff is removed. A related possibility that we will explore in future research is to introduce a dynamical cutoff, dependent on the value of $v_{\theta}$, as an effective way to account for the minimum coordinate length $\bar{\mu}_{\theta}$.

Other situations in which the structure of the solutions is well under control is when one regards the inhomogeneous part of the Hamiltonian constraint as a perturbation around Bianchi I and truncates the solutions at second perturbative order in it or, alternatively, if one views as a perturbation only the term that contains $\hat{H}_{\text {int }}^{\xi}$ and makes the truncation at first order. Then the solutions can be defined again, up to the analyzed perturbative order, in the dual of the $n$-particle states for each $v_{\theta}$ section and the physical Hilbert space that one obtains is just $L^{2}\left(\mathbb{R}^{2}, d \lambda_{\sigma} d \lambda_{\delta}\right) \otimes \mathcal{F}_{p}$.

Note that in the above cases we can adopt the picture that solutions are initial data on the section $v_{\theta}=\varepsilon_{\theta}$ which evolve to new data on any other section $v_{\theta}=\varepsilon_{\theta}+2 k$. Identifying sections, we arrive in principle at a concept of evolution for physical states which would not be unitary (i.e. the norm would not be preserved) as a consequence of the polymeric quantization of the internal time.

Furthermore, the loop quantization carried out on the homogeneous sector allows us to get insight about the resolution of the cosmological singularity. The classical Gowdy model generically presents an initial singularity where some of the variables $p_{i}$ vanish. Since zero-volume states are removed in the quantum theory, the kernel of any of the operators $\hat{p}_{i}$ is empty. Therefore, the singularity disappears already at the kinematical level, and, at least in this sense, is resolved. Note that, in a standard quantization of the homogeneous sector, the operators $\hat{p}_{i}$ have instead a continuous spectrum. Thus, their kernel is not a proper subspace which could be decoupled in order to remove the singularity in the kinematical Hilbert space. Moreover, the singularity then persists also in the physical Hilbert space. This fact can be easily realized in the completely deparametrized system quantized à la Fock [12], since the domain of definition of the time parameter plays there the role of the spectrum of $\left|\hat{p}_{\theta}\right|$ quantized in the standard way. Curvature invariants become explicitly timedependent quantum observables that indeed diverge in the limit when the time parameter vanishes [9].

To conclude, we have successfully quantized this inhomogeneous Gowdy model by combining loop and Fock quantizations. The attained results prove that our hybrid quantization is feasible and well posed. More precisely, we have represented rigorously the quantum constraints and explicitly found their solutions, which have been endowed with a Hilbert structure. The physical Hilbert space thus obtained reproduces the conventional Fock quantization of the inhomogeneous modes. As far as we know, this is the first quantization carried out to this degree of completion in an inhomogeneous cosmological system using polymeric techniques. The loop quantization of the homogeneous 
sector proves enough to resolve the classical singularity, inasmuch as it disappears from the quantum theory. In fact, it is not only avoided in physical states, but they never cross it. The quantum states of our Gowdy universe start in a single nonsingular section, without requiring any boundary conditions. This result reinforces the robustness of the bigbang avoidance in LQC and paves the road for the extension of the singularity resolution to inhomogeneous cases.
The authors are very grateful to J. M. Velhinho and T. Pawlowski. This work was supported by the Spanish Grants No. FIS2005-05736-C03-02 (its continuation No. FIS2008-06078-C03-03), No. FIS2006-26387-E, and No. CSD2007-00042 (CPAN); and M. M-B. by CSIC and the European Social Fund under the Grant No. I3PBPD2006.
[1] M. Bojowald, Living Rev. Relativity 11, 4 (2008).

[2] T. Thiemann, Modern Canonical Quantum General Relativity (Cambridge University Press, Cambridge, England, 2007).

[3] A. Ashtekar, M. Bojowald, and J. Lewandowski, Adv. Theor. Math. Phys. 7, 233 (2003).

[4] See, e.g., A. Ashtekar, T. Pawlowski, and P. Singh, Phys. Rev. Lett. 96, 141301 (2006); Phys. Rev. D 73, 124038 (2006); 74, 084003 (2006).

[5] D. W. Chiou, Phys. Rev. D 75, 024029 (2007).

[6] D. W. Chiou, Phys. Rev. D 76, 124037 (2007).

[7] K. Banerjee and G. Date, Classical Quantum Gravity 25, 145004 (2008).

[8] R. H. Gowdy, Ann. Phys. (N.Y.) 83, 203 (1974).

[9] V. Moncrief, Phys. Rev. D 23, 312 (1981).

[10] See, e.g., C. W. Misner, Phys. Rev. D 8, 3271 (1973); B. K. Berger, Ann. Phys. (N.Y.) 83, 458 (1974); Phys. Rev. D 11, 2770 (1975); Ann. Phys. (N.Y.) 156, 155 (1984); G. A. Mena Marugán, Phys. Rev. D 56, 908 (1997); M. Pierri, Int. J. Mod. Phys. D 11, 135 (2002).

[11] See, e.g., K. Kuchar, Phys. Rev. D 4, 955 (1971); A. Ashtekar and M. Pierri, J. Math. Phys. (N.Y.) 37, 6250 (1996); J.F. Barbero G., G. A. Marugán, and E.J.S.
Villaseñor, Phys. Rev. D 67, 124006 (2003).

[12] A. Corichi, J. Cortez, and G. A. Mena Marugán, Phys. Rev. D 73, 041502 (2006); 73, 084020 (2006); A. Corichi, J. Cortez, G. A. Mena Marugán, and J.M. Velhinho, Classical Quantum Gravity 23, 6301 (2006); J. Cortez, G. A. Mena Marugán, and J. M. Velhinho, Phys. Rev. D 75, 084027 (2007).

[13] M. Bojowald, Phys. Rev. Lett. 86, 5227 (2001).

[14] V. A. Belinsky, I. M. Khalatnikov, and E. M. Lifshitz, Adv. Phys. 31, 639 (1982).

[15] We will not use the Einstein summation convention.

[16] There exist different proposals to define this minimum value, which are still under discussion. This issue has been considered in [6] and by A. Ashtekar (unpublished).

[17] M. Martín-Benito, G. A. Mena Marugán, and T. Pawlowski, Phys. Rev. D 78, 064008 (2008).

[18] M. Martín-Benito, Master thesis, Universidad Complutense de Madrid, 2007.

[19] Namely, the algebraic dual of the subset of $\mathrm{Cyl}_{S}$ of nonzero-volume states.

[20] L. J. Garay, M. Martín-Benito, G. A. Mena Marugán, and J. M. Velhinho (unpublished). 